\title{
Forschen zum Wohl der Patienten
}

Die starke Zunahme des Diabetes in der alternden Bevölkerung bringt das deutsche Gesundheitssystem an die Grenzen seiner Belastbarkeit. Umso wichtiger ist es, durch neue wissenschaftliche Ergebnisse wirksame Präventions- und Behandlungsmaßnahmen zu entwickeln und so der Entstehung bzw. dem Fortschreiten des Diabetes Einhalt zu gebieten.

Wichtige Fragen in der Diabetesforschung sind: Wie entsteht die Krankheit, welche Faktoren tragen dazu bei? Wie kann man sie verhindern, therapieren oder gar heilen? Die Antworten kann kein einzelner Wissenschaftler, keine Arbeitsgruppe, keine Institution alleine finden. Nur ein integrativer Forschungsansatz, der die verschiedenen Forschungsdisziplinen vereint, vermag das komplexe Geschehen zu entschlüsseln.

Um zukünftig Forschungsergebnisse schneller in die klinische Praxis zu bringen, hat die Bundesregierung 2009 das Deutsche Zentrum für Diabetesforschung (DZD) als eines von insgesamt 6 Deutschen Zentren der Gesundheitsforschung gegründet.

Partner im DZD sind das Helmholtz Zentrum München, das Deutsche Diabetes-Zentrum in Düsseldorf, das Deutsche Institut für Ernährungsforschung PotsdamRehbrücke und die Universitäten Tübingen und Dresden. Ziel des Forschungsverbundes ist es, durch einen engen Austausch von Grundlagenforschern und klinisch tätigen Ärzten, neue personalisierte Präventionsstrategien und Therapien des Diabetes zu entwickeln. Diese sollen zum Wohl der Patienten möglichst zeitnah den Weg in die Arztpraxen und Kliniken finden.

Die DZD-Partnerinstitute und ihre Forschungsbereiche sind über eine Vielzahl an Projekten exzellent vernetzt. Auf den nächsten Seiten finden Sie einen Überblick über die Forschungsschwerpunkte und Strukturen des DZD.

Dr. Bettina Nowotny beschreibt mit der Deutschen Diabetes-Studie langjährige Beobachtungsstudie, an der Diabetespatienten teilnehmen. Ziel ist es, die Entstehungsmechanismen des Diabetes besser zu verstehen. Dadurch erhoffen sich die DZD-Wissenschaftler zukünftig eine bessere Prognose des Krankheitsverlaufs, wodurch der Arzt frühzeitig durch eine geeignete Therapie gegensteuern kann.

Einblicke in die diagnostische Praxis entsprechend den neuen Leitlinien gewährt Prof. Erwin Schleicher. Er stellt die Neuerungen durch Zulassung des $\mathrm{HbA}_{1 \mathrm{c}}$-Wer- tes für die Labordiagnostik des Diabetes mellitus in den Mittelpunkt.

Mit dem Deutschen Diabetes-Risiko-Test ${ }^{\circledR}$ beschreiben Prof. Matthias Schulze und Prof. Annette Peters die erfolgreiche praktische Anwendung epidemiologischer Forschungsergebnisse. Darüber hinaus thematisieren die Autoren Untersuchungen des DZD, die die Überlegenheit der Aussagekraft des BMI (Body Mass Index) im Vergleich zum jüngst entwickelten BAI (Body Adiposity Index) bestätigen.

Dr. Harald Grallert und seine Kollegen Dr. Timo Kanzleiter und Dr. Robert Schwenk erklären mit der Genetik und Epigenetik des Typ-2-Diabetes einen möglichen Schlüssel zur personalisierten Medizin. Neben dem ererbten genetischen Code stehen insbesondere durch den Lebensstil erworbene Veränderungen der Genregulation zusammengefasst - unter dem Begriff Epigenetik - im Fokus der genetischen Forschung. Durch ein tieferes Verständnis dieser Mechanismen versprechen sich die Wissenschaftler des DZD zukünftig eine spezifischere Diagnostik, die eine feinere Einteilung der Erkrankung in Subklassen ermöglicht. Dadurch sollen Prädiktion, Prävention und insbesondere die individualisierte Therapie von Diabetespatienten verbessert werden.

Dem metabolischen Fingerabdruck sind PD Dr. Philippe Schmitt-Kopplin und seine Mitautoren mithilfe von Metabolomics - der Detektion von Metaboliten in biologischen Proben - auf der Spur. Sie stellen Ihnen diese neue Technologie und ihre Anwendung in der Diabetesforschung in diesem Heft vor.

Nach dem Motto „Jeder soll wissen, dass ein gesunder Lebensstil das Auftreten eines Typ-2-Diabetes verzögern oder gar verhindern kann“ liegt es uns DZD-Sprechern sehr am Herzen, unsere Ergebnisse Ärzten, medizinischem Fachpersonal, aber auch der Bevölkerung zugänglich zu machen.

Medizin der Zukunft bedeutet, die Entstehung des Diabetes durch eine maßgeschneiderte Prävention zu verhindern bzw. dem Patienten eine individuelle kausale Therapie zum richtigen Zeitpunkt zur Verfügung zu stellen. Auf dem Gebiet des Diabetes sind wir von diesem anspruchsvollen Ziel noch weit entfernt. Mit unserer Mission „Forschen für eine Zukunft ohne Diabetes“ stellen wir uns im DZD den wissenschaftlichen Herausforderungen.

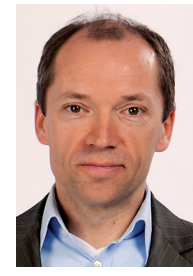

Prof. Dr. Martin Hrabé de Angelis

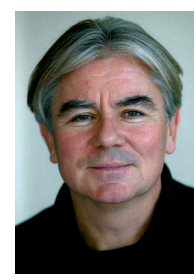

Prof. Dr. Dr. h.c. Hans-Ulrich Häring

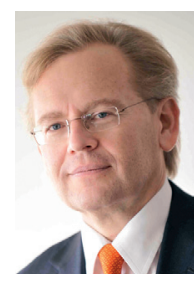

Prof. Dr. Michael Roden

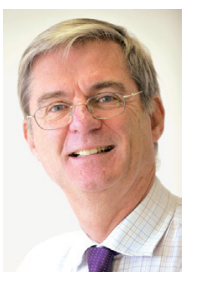

Prof. Dr. Dr. Hans-Georg Joost

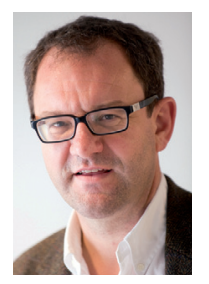

Prof. Dr. Michele Solimena 